Revistade
Economila
Contemporâned

\title{
POLÍTICA MONETÁRIA E MERCADO DE TRABALHO NO BRASIL
}

\author{
Michel Cândido de Souza ${ }^{a}$ \\ ${ }^{a}$ Estudante do doutorado em Economia no Centro de Desenvolvimento e Planejamento \\ Regional da Universidade Federal de Minas Gerais (CEDEPLAR/UFMG)
}

Artigo recebido em 13/05/2016 e aprovado em 03/05/2017.

\begin{abstract}
RESUMO: A partir de um modelo dinâmico estocástico de equilíbrio geral (DSGE), seguindo Christoffel, Kuester e Linzert(2009) com fricções do tipo searching and matching, o presente estudo busca verificar os efeitos de um choque de política monetária negativo sobre o mercado de trabalho brasileiro. As simulações apontam que o choque leva ao aquecimento do consumo interno, aumento das contratações por parte das firmas e elevação do salário após o processo de barganha. No entanto, o mesmo choque reduz a probabilidade de novas vagas serem preenchidas, já que o aumento do número de matches é menor do que o aumento de vagas disponíveis, o que dificulta uma queda mais acentuada no desemprego. Por fim, a comparação dos desvios padrão e correlações do modelo com os dados aponta para indícios de um possível processo de rigidez salarial forte no país, dificultando os ganhos do trabalhador.
\end{abstract}

PALAVRAS-CHAVE: política monetária; mercado de trabalho; searching and matching; DSGE.

CLASSIFICAÇÃO JEL: E12, E24, E52.

Correspondência para: Michel Cândido de Souza

Contato: michel.cands@gmail.com 


\title{
MONETARY POLICY AND LABOR MARKET IN BRAZIL
}

\begin{abstract}
From a dynamic stochastic general equilibrium (DSGE) model, following Christoffel, Kuester and Linzert (2009), with searching and matching frictions, this study aims to verify the effects of a negative monetary policy shock on the Brazilian labor market. The simulations show that the shock leads to heating of domestic consumption, increased hiring by firms and raising the wage after the bargaining process. However, the same schock reduces the probability of new vacancies be filled, as the increase in the number of matches is less than the increase in available positions, which hinders a sharper fall in unemployment. Finally, the comparison of model standard deviations and correlations to the data points to evidence of a possible strong wage rigity process in the country, hindering the employee's earnings.
\end{abstract}

KEYWORDS: monetary policy; labor market; searching and matching; DSGE. 


\section{INTRODUÇÃO}

O mercado de trabalho afeta diretamente as oscilações de outras variáveis macroeconômicas, sendo um dos grandes motores das economias modernas. Sua boa organização e a eficiência com que as transações empregatícias ocorrem são fatores impactantes no processo produtivo de um país. Ou seja, o tempo de procura por um posto de trabalho, a probabilidade de uma nova vaga ser preenchida, a destruição de vagas disponíveis, a combinação entre a procura e a oferta das vagas (matching), entre outros, estão fortemente relacionados ao desemprego e à produção agregada.

Os modelos Stochastic General Equilibrium (DSGE) podem ser utilizados na elaboração de análises de sensibilidade sobre o emprego agregado. Essa metodologia, em resumo, representa uma aplicação da teoria de equilíbrio geral, na tentativa de mensurar oscilações macroeconômicas em resposta a choques estocásticos exógenos (como na produtividade, política monetária, política fiscal, entre outros), dado o comportamento intertemporal de consumidores e firmas dentro da economia. A crescente adoção desses modelos tem sido atribuída, em grande parte, aos insights gerados no debate de políticas monetárias ótimas, fomentados majoritariamente pelos Bancos Centrais em todo o mundo. Como exemplo, o Banco Central do Brasil (BACEN) já desenvolve, há anos, um modelo DSGE próprio para a análise monetária, denominado Stochastic Analytical Model with a Bayesian Approach (SAMBA). No entanto, a modelagem DSGE para o Brasil ainda é escassa, principalmente no que se refere à análise de como fricções no mercado de trabalho podem ajudar a compreender oscilações econômicas.

O foco deste estudo é analisar a interação entre a política monetária e a dinâmica do mercado de trabalho no Brasil. Buscou-se investigar se a introdução de fricções no mercado de trabalho brasileiro ajuda a compreender melhor o desvio padrão das séries históricas. Os principais objetivos específicos deste trabalho são: i) contribuir com a literatura nacional, apresentando a aplicação de um modelo DSGE desenvolvido por Christoffel, Kuester e Linzert (2009); ii) calibrar detalhadamente o modelo, com fricções do tipo searching and matching, para a realidade da economia brasileira; iii) verificar o ajustamento do modelo em relação às séries históricas de produto e desemprego; iv) elaborar uma análise de impulso resposta sobre o equilíbrio econômico no país, dado um choque de política monetária negativo (ou redução da taxa de juros nominal).

\section{FRICÇÕES NO MERCADO DE TRABALHO}

A hipótese de que em um mercado qualquer compradores e vendedores se encontram de forma não custosa e detêm informações perfeitas em relação aos preços e à proce- 
dência dos bens ofertados, foi adotada por muito tempo no processo de desenvolvimento de grandes teorias clássicas do desemprego.

Já na década de 1980, alguns pesquisadores como Diamond (1982), Mortensen (1982) e Pissarides (1985) passaram a buscar flexibilizações dessa teoria, investigando profundamente a existência de fricções no mercado de trabalho. O desenvolvimento da chamada função match, que capta a taxa com que trabalhadores desempregados e firmas com vagas disponíveis se encontram, foi uma das alternativas que emergiram em relação à abordagem clássica. A proposta buscava explicar as altas taxas de desocupação presentes nas grandes economias do período, assumindo a existência de desemprego e vagas ociosas em um mesmo modelo econômico.

Similar a uma função de produção comum, com formato Cobb-Douglas, a função match possui duas entradas: desempregados e vagas ociosas. O produto dessa função são os novos matches que ocorrem nos setores produtivos da economia ao longo dos períodos. De um lado, os agentes desempregados buscam por postos de trabalho que proporcionem retorno superior ao salário reserva. No oposto, as firmas buscam por trabalhadores que preencham as vagas ociosas e proporcionem retornos superiores aos custos envolvidos no processo de contratação. Quando existe acordo entre as partes, o trabalhador se dispõe a iniciar sua jornada por um salário considerado por ele justo, a empresa prevê um retorno superior ao custo marginal do trabalho, o setor produtivo recebe uma nova participação, o search tem êxito e o match ocorre.

Como destacamos, as contribuições pioneiras dessa literatura foram desenvolvidas por Diamond (1982a), Diamond (1982b), Mortensen (1982), Pissarides (1985) e Mortensen e Pissarides (1994), laureados com prêmio Nobel em 2010 pela abordagem conhecida como DMP. Diamond (1982a, 1982b) introduz a existência de tecnologia imperfeita para busca de vagas no mercado de trabalho. Mostrando que o desemprego friccional ocorre justamente a partir de um movimento condicional dos salários em relação ao nível de desocupação e a criação de novas vagas no mercado, dada a tecnologia de informação vigente. Mortensen (1982) segue na mesma abordagem searching and matching, mas contribui pela análise do mercado de trabalho como um jogo não cooperativo. $\mathrm{O}$ autor destaca a importância dos efeitos do poder de barganha sobre o nível de novas combinações no mercado de trabalho e a dinâmica de incentivos que os agentes desempregados possuem para investirem em processos que facilitem o match (como contatos, informação, qualificação, entre outros). Em sequência, Pissarides (1985) apresenta consideráveis avanços na análise de resposta do desemprego, salários e vagas em relação a choques aplicados sobre o valor real do produto, indicando que essas variáveis apresentam comportamento assimétrico. Isso ocorre porque a velocidade de resposta do desemprego é mais rápida para choques negativos do que para positivos no produto, ou seja, períodos de recessão tendem a intensificar o aumento 
do desemprego. Finalmente, Mortensen e Pissarides (1994) incluem a possibilidade de destruição dos postos de trabalho nos modelos. A simples criação e destruição de postos de trabalho é de grande importância, pois os custos que esses movimentos geram determinam o equilíbrio do modelo, já que afetam diretamente as oportunidades de matching dos agentes.

Em síntese, esses autores ressaltam dois pontos fundamentais que geram parte do desemprego nas economias: i) dinâmica imperfeita do match - existe falta de coordenação entre os agentes participantes do mercado de contratações, de forma que nem todos os desempregados são capazes de encontrar uma vaga e vice-versa; ii) custos empregatícios - preencher uma vaga é um procedimento demorado e caro para ambas as partes. Além disso, o salário é um componente resultante da negociação entre empresas e trabalhadores, na qual estes últimos possuem algum grau de poder de barganha na negociação e podem elevar o salário acima do nível socialmente desejável.

Após essas contribuições, a aplicabilidade da função searching and matching foi incorporada em modelos DSGE, tanto nos clássicos do tipo Real Business Cycles quanto nos modelos novo-keynesianos. Em geral, os resultados mostraram que a inclusão do ambiente search no mercado de trabalho representa um importante mecanismo de propagação dos choques, tornando o equilíbrio searching encontrado consistente com um número considerável de fatos empíricos estilizados da economia norteamericana. Destacamos os trabalhos de Andolfatto (1996), Denhann e Watson (2000), Petrongolo e Pissarides (2001), Shimer e Smith (2001), Hall (2005), Mayer, Moyen e Stahler (2010) e Kato e Miyamoto (2015).

A literatura brasileira é escassa em relação a pesquisas que abordem as fricções do mercado de trabalho em modelos DSGE. Dois trabalhos recentes que se aprofundam nessa discussão merecem destaque: Basílio (2012) e Attuy (2012). No trabalho de Basílio (2012), o autor desenvolve um modelo de equilíbrio geral novo-keynesiano, no qual firmas formadoras de preços interagem com a autoridade monetária. Partindo dessa ligação, demonstra que as interações entre a autoridade monetária e as instituições do mercado de trabalho, representadas matematicamente por funções de poder de barganha sindical, geram um efeito Calmfors-Driffill na economia brasileira. Esse efeito mostra que diferentes níveis de centralização sindical implicam em resultados econômicos distintos, mas em geral a centralização tende a reduzir a abertura de vagas por parte das firmas formadoras de preço, uma vez que afeta diretamente o salário dos trabalhadores, exercendo efeito quadrático sobre o desemprego. Basílio (2012) sugere que um maior conservadorismo na condução da política monetária garante melhores resultados em termos de desemprego e inflação, dada a modelagem do poder de barganha dos sindicatos. Ademais, o autor foca a análise sobre os efeitos que o poder de negociação dos sindicatos exerce diretamente sobre os salários na economia 
e indiretamente sobre as demais variáveis reais, não se preocupando com a análise do fenômeno matching.

Attuy (2012), por sua vez, trabalha com duas vertentes aplicadas ao caso brasileiro: um modelo básico RBC com fricções do tipo DMP e um modelo novo-keynesiano, com rigidez nominal, indexação de preços, reajuste dos salários, preços à la Calvo, custo de ajustamento para investimento das famílias e persistência de hábito de consumo. As novidades introduzidas são em relação aos trabalhadores desempregados involuntariamente pela própria dinâmica de match e ao tratamento da taxa de desemprego como componente central da economia.

O autor define de forma heterogênea, e empírica, a taxa de separação dos trabalhadores brasileiros, utilizando o valor estimado como parâmetro para calibração no modelo RBC com fricções DMP. Em seguida, estima o modelo novo-keynesiano para economia brasileira, buscando identificar quais são as fricções do mercado de trabalho mais importantes na oscilação das variáveis macroeconômicas, verificar o comportamento específico da série de desemprego dentro da modelagem e discutir o efeito dos choques sobre a taxa de desemprego.

Os resultados apontam que a inserção de todas as fricções (exceto de indexação salarial) melhora o ajustamento de ambos os modelos aos dados analisados. Sendo que as fricções reais aparentam maior importância quando a taxa de desemprego é trabalhada no lugar do valor agregado. Por fim as funções impulso resposta, dado um choque de política monetária expansionista, indicam que: o consumo interno cai, o desemprego aumenta sendo puxado pela queda no número de matches e a inflação é reduzida pelo aumento dos juros.

A partir do que foi exposto nesta seção, as fricções do mercado de trabalho, especialmente no tipo search and matching, aparentam ser um componente desafiador e relevante na modelagem matemática das variações econômicas. A próxima seção se dedica à exposição completa do modelo de estudo, baseado na obra de Christofell, Kuester e Linzert (2009), em seguida, na seção 4, os resultados são apresentados, e na seção 5 as conclusões são destacadas.

\section{MODELO DE ESTUDO}

As análises desenvolvidas no presente estudo são baseadas em um modelo calibrado para o Brasil cuja abordagem estrutural utilizada é encontrada no trabalho de Christoffel, Kuester e Linzert (2009).Este segue uma modelagem novo-keynesiana com search and match para o mercado de trabalho e estrutura right-to-manage nos salários, permitindo um canal de conexão direto entre salários e inflação, no qual trabalhadores 
e empresas barganham somente sobre a taxa de salário por hora e as empresa escolhem a quantidade de emprego posteriormente. Além disso, firmas e trabalhadores negociam em um ambiente com rigidez à la Calvo (1983), com custos fixos relacionados ao trabalho.

\subsection{FAMÍLIAS}

Os consumidores representativos ofertam horas de trabalho e demandam bens finais produzidos pelas firmas. O operador $E\left[\right.$.] denota expectativas, $c_{i, t}$ é o consumo e $h_{i, t} \mathrm{o}$ número de horas trabalhadas. O parâmetro $\beta \in(0,1)$ indica o fator de desconto subjetivo da economia; $\kappa^{L}$ é a desutilidade gerada pelo trabalho na definição das preferências; $\sigma$ representa a aversão relativa ao risco do consumidor; $\varphi$ é a elasticidade da oferta de horas de trabalho; e $\rho$ representa o parâmetro de hábito de consumo. Dessa forma, a função utilidade, que reflete as preferências do consumidor, é dada por:

$$
E \sum_{t=0}^{\infty} \beta^{t} \frac{\left(c_{i, t}-\rho c_{i, t-1}\right)^{1-\sigma}}{1-\sigma}-\kappa^{L} \frac{\left(h_{i, t}\right)^{1+\phi}}{1+\phi}
$$

A equação (1) mostra que os agentes obtêm satisfação ao consumir, porém quanto maior o número de horas trabalhadas, menor a utilidade, devido à redução direta do tempo de lazer. Em sequência, existe um continuum de famílias idênticas que vivem em um horizonte de tempo infinito, compostas por uma proporção de $n_{t}=1-u_{t}$ membros empregados e $u_{t}$ membros desempregados, ambos com preferências conforme as retratadas acima. As famílias escolhem consumir e poupar em quantidades que maximizam a função utilidade, no entanto, existe uma restrição orçamentária vigente na economia tal que: $c_{t}$ representa o consumo agregado das famílias; $t_{t}$, o imposto lump-sum pago; $\kappa v_{t}$ é o custo agregado proveniente da abertura de novas vagas ${ }^{1} ; W_{i, t} h_{i, t}$ indica a receita advinda do trabalho e $P_{t}$, o nível de preços da economia; $D_{t}$ representa os títulos livres de risco; $R_{t}$, a taxa nominal de juros e $u_{t} b$, o benefício do desemprego pago pelo governo, no formato puro de transferências de renda. Em relação à parte financeira, as famílias recebem $\Phi^{K}$ de renda advinda do capital e possuem ações de todas as empresas presentes na economia, as quais pagam $\Psi_{t}$ de receita real de dividendos.

$$
c_{t}+t_{t}+\kappa v_{t}=\int_{0}^{1-u_{t}} W_{i, t} h_{i, t} d i+u_{t} b+\frac{D_{t-1} R_{t-1}}{P_{t}}-\frac{D_{t}}{P_{t}}+\Psi_{t}+n_{t} \Phi^{K} \sum
$$

\footnotetext{
1 Aqui o custo de abertura de uma nova vaga é arcado pelas famílias, pois o modelo pressupõe que as mesmas são donas das firmas.
} 
Como não há presença explícita de capital, supõe-se que as famílias ao mesmo tempo ofertam e recebem uma renda fixa proveniente deste fator, dada por $n_{t} \Phi^{K}$. A receita de dividendos $\Psi_{t}$ é dada pela soma de $\Psi_{t}^{C}$ e $\int_{0}^{1-u_{t}} \Psi_{i, t}{ }^{L} d i$, que são os lucros gerados no setor das indústrias de diferenciação de produtos e nas empresas de bens de trabalho, respectivamente.

\subsection{MERCADO DE TRABALHO}

Seguindo Mortensen e Pissarides (1994) e Andolfatto (1996), as fricções no mercado de trabalho são representadas por uma função matching, no formato Cobb-Douglas. Essa função resume uma tecnologia de negociação vigente entre agentes que colocam anúncios, leem jornais e revistas, vão a agências de emprego e utilizam contatos para sua alocação no mercado de trabalho. A expressão abaixo mostra que o número de novos matchs produzidos, $m_{t}$, depende da quantidade de trabalhadores que buscam uma nova ocupação (taxa de desempregados), $u_{t}=1-n_{t}$, e do número de vagas disponíveis no mercado de trabalho, $v_{t}$. Já $\xi$ e $\sigma_{m}$ representam a elasticidade dos insumos na função e o parâmetro de eficiência global do matching, respectivamente.

$$
m_{t}\left(v_{t}, u_{t}\right)=\sigma_{m} u_{t}^{\xi} v_{t}^{1-\xi}
$$

Essa escolha funcional deve-se à característica moderna do processo de busca por ocupações, que necessita de tempo e recursos dos agentes econômicos, considerando que apenas uma parcela dos que procuram pela sua contraparte obtem sucesso. Além disso, nesse modelo todas as famílias possuem igual probabilidade de encontrar ou perder um emprego. Essa abordagem advém do modelo de Pissarides (1985), que exclui movimentos de dentro e fora do mercado de trabalho na definição dos parâmetros estruturais, ou seja, a elasticidade e a eficiência do matching não ficam condicionados a oscilações de curto prazo.

Devido à homogeneidade linear de grau um da função matching, a probabilidade de uma vaga anunciada ser preenchida $q_{t}$ e a probabilidade de um agente encontrar emprego $s_{t}$ são definidas como:

$$
\begin{gathered}
q_{t}=\frac{m_{t}}{v_{t}} \\
s_{t}=\frac{m_{t}}{u_{t}}
\end{gathered}
$$


O mercado de trabalho possui uma dinâmica própria: a proporção de trabalhadores ativos é dada pela soma de $n_{t-1}$ trabalhadores que não foram demitidos no período anterior (de acordo com a probabilidade $\vartheta_{t}$ do trabalhador não se manter empregado no período sequente) e $m_{t-1}$ novos matches que se tornaram produtivos em $t$.

$$
n_{t}=\left(1-\vartheta_{t}\right) n_{t-1}+m_{t-1}
$$

\subsection{FIRMAS}

Existem três tipos de firmas nesta economia: firmas de bens de trabalho, firmas atacadistas e firmas de varejo. As empresas que produzem bens intermediários homogêneos, ou bens de trabalho, precisam encontrar exatamente um trabalhador para produção e em sequência vendem ao setor atacadista, por meio de um mercado competitivo. Já as empresas atacadistas tomam o bem de trabalho como insumo necessário para produção de bens diferenciados, a partir de uma tecnologia de retornos constantes de escala, e ficam sujeitas a impedimentos de escolhas de preços, além de lidar com rigidez à la Calvo e concorrer de forma monopolística na venda dos produtos diferenciados para setor final de varejo. Finalmente, as firmas varejistas agregam produtos diferenciados em uma cesta de consumo homogênea, $y_{t}$, e vendem como bem final ao preço $P_{t}$ para os consumidores e governo.

\subsubsection{FIRMAS DE VAREJO}

Definindo um bem de consumo final como $y_{t}$, o qual é produzido por uma cesta de bens do setor atacadista, e um bem de atacado específico do tipo $j$ como $y_{j, t} \forall j \in[0,1]$, temos:

$$
y_{t}=\left(\int_{0}^{1} y_{j, t}^{\frac{\varepsilon-1}{\varepsilon}} d j\right)^{\frac{\varepsilon}{\varepsilon-1}}
$$

Em (7), $\varepsilon>1$ é a elasticidade da substituição entre os produtos diferenciados. A solução do problema de minimização do dispêndio implica que a demanda pelo bem de atacado do tipo $j$ é inversamente relacionado com o preço relativo de varejo do tipo $j$. Assumindo competição perfeita e lucro zero no setor de agregação, o índice de preços $P_{t}$ fica relacionado aos preços individuais $P_{j, t}$ através da seguinte sentença:

$$
P_{t}=\left(\int_{0}^{1} P_{j, t}^{1-\varepsilon}\right)^{\frac{1}{1-\varepsilon}}
$$




\subsubsection{FIRMAS ATACADISTAS}

As empresas do setor atacadista são indexadas por $j \in[0,1]$ e produzem uma variedade $j$ de bens diferenciados. Aqui $l_{j, t}^{d} v$ denota a demanda pelo bem de trabalho intermediário, que a empresa de atacado $j$ pode adquirir em um mercado perfeitamente competitivo pelo preço real $x_{t}^{L}$.

$$
y_{j, t}=l_{j, t}^{d}
$$

Os lucros reais, por período, da empresa $j$ são dados por:

$$
\Psi_{j, t}^{C}=\frac{P_{j, t}}{P_{t}} y_{j, t}-l_{j, t}^{d} x_{t}^{L}
$$

O primeiro termo mostra as receitas do atacado e o segundo o custo dos bens de trabalho. Em sequência, em cada período uma fração aleatória $\omega \in[0,1]$ das empresas atacadistas não pode reajustar seu preço; estas indexam seu preço à taxa de inflação do último período, $\Pi_{t-1}$, e a taxa de inflação de estado estacionário $\Pi$. O fator de indexação inflacionária é modelado como $\Pi_{t-1}^{\varepsilon_{\mathrm{p}}} \Pi^{1-\varepsilon_{\mathrm{p}}}$ com grau de indexação relativo aos preços passados dado por $\varepsilon_{\mathrm{p}} \in[0,1]$.

Já as empresas que reajustam seu preço no período $t$ enfrentam o problema de maximização do seu valor escolhendo o preço de venda $P_{j, t}$, tendo em conta as fricções, a função de demanda e a função de produção. Abaixo, o termo entre parênteses no numerador representa o mecanismo de indexação parcial; $m_{c t}$ se refere ao custo marginal real tal que $m_{c t}=x_{t}^{L}$ e $\beta_{t, t+s}$ é o fator desconto estocástico de equilíbrio. Destacando que, para qualquer demanda, a escolha ótima de insumos do trabalho leva a um custo marginal que independe da quantidade produzida.

$$
\max _{P_{j, t}} E_{t}\left\{\sum_{s=0}^{\infty} \omega^{s} \beta_{t, t+s}\left[\frac{P_{j, t}\left(\Pi_{t-1, t-1+s}^{\varepsilon_{p}}\left(\Pi^{1-\varepsilon} p\right)^{s}\right)}{P_{t+s}}-m c_{t+s}\right] y_{j, t+s}\right\}
$$

O total de lucros reais do setor é definido como $\Psi_{t}^{C}=\int_{0}^{1} \Psi_{j, t}^{C} d j$.

\subsubsection{FIRMAS DE BENS DE TRABALHO}

Os bens de trabalho são homogêneos e as empresas nesse setor precisam encontrar exatamente um trabalhador a fim de produzir. Em cada período $t$ existem $1-u_{t}$ empresas de bens de trabalho operando. Considerando $\alpha \in(0,1)$ como a elasticidade trabalho e 
$z_{t}$ como a produtividade, o match na empresa $i$ pode produzir uma quantidade $l_{i, t}$ do bem de trabalho, utilizando horas trabalhadas $h_{i, t}$, de acordo com a seguinte equação:

$$
l_{i, t}=z_{t} h_{i, t}^{\alpha}
$$

\subsection{FUNÇÃO VALOR DOS TRABALHADORES}

Devido às fricções e aos retornos decrescentes de escala, para firma de bens de trabalho, a formação de matches implica em renda econômica. Nesse modelo, empresas e trabalhadores entram em um processo de barganha pela maior participação no excedente de match. Seguindo Denhaan e Watson (2000), as famílias decidem o quanto os membros ofertam de trabalho. Um trabalhador empregado obtém a renda do salário, mas sofre o custo de utilidade do trabalho. No período seguinte, o trabalhador fica desempregado com probabilidade $\vartheta_{t}$ ou mantém o seu trabalho com probabilidade $\left(1-\vartheta_{t}\right)$. Se fica empregado em $t+1$, com probabilidade $\gamma$, não será apto a entrar em um novo processo de barganha do seu salário nominal. No caso do trabalhador que não pode renegociar, o salário nominal é parcialmente indexado à inflação pela proporção $\xi_{w}$. Mas se o trabalhador for capaz de entrar em um novo processo de barganha, seu valor reflete o salário ótimo renegociado em $t+1$. Sendo assim, o valor (para a família) de um trabalhador $V_{t}^{E}$ que está empregado e recebe salário nominal $W_{i, t}$ é:

$$
\begin{aligned}
& V_{t}^{E}\left(W_{i, t}\right)=\frac{W_{i, t}}{P_{t}} h_{i, t}-\frac{\kappa^{L} h_{i, t}^{1+\phi}}{\lambda_{t}(1+\phi)} \\
& +E_{t}\left\{\beta_{t, t+1}\left(1-\vartheta_{t+1}\right)\left[\gamma V_{t+1}^{E}\left(W_{i, t}\left[\Pi_{t}^{\varepsilon_{w}} \Pi^{1-\varepsilon_{w}}\right]\right)+(1-\gamma) V_{t+1}^{E}\left(W_{t+1}^{*}\right)\right]\right\}+E_{t}\left\{\beta_{t, t+1} \vartheta_{t+1} V_{t+1}^{U}\right\}
\end{aligned}
$$

Como destacado acima, com probabilidade $\vartheta_{t}$ o trabalhador se encontra desempregado no próximo período, dessa forma o valor (para a família) de ter um trabalhador que está desempregado é dado, teoricamente, pela próxima equação abaixo, onde $b$ representa o benefício concedido pelo governo aos trabalhadores desempregados. Um trabalhador que está desempregado em $t$ tem probabilidade $s_{t}$ de encontrar um novo emprego e será produtivo a partir de $t+1$. Novamente, com probabilidade $(1-\gamma)$ os trabalhadores da família podem negociar o salário em $t+1$ e com probabilidade $\gamma$ começam a trabalhar a um salário/hora condizente com a média dos salários já existentes em $t$, parcialmente indexado à inflação:

$$
V_{t}^{U}\left(W_{t}\right)=b+E_{t}\left\{\beta_{t, t+1} S_{t}\left[\gamma V_{t+1}^{E}\left(W_{t}\left[\Pi_{t}^{\varepsilon_{w}} \Pi^{1-\varepsilon_{w}}\right]\right)+(1-\gamma) V_{t+1}^{E}\left(W_{t+1}^{*}\right)\right]\right\}+E_{t}\left\{\beta_{t, t+1}\left(1-s_{t}\right) V_{t+1}^{U}(W)_{t}\right\}
$$


Isso implica rigidez no salário por hora de novos matches. A lógica dessa modelagem é que como as empresas têm muitos postos de trabalho, estes podem ser preenchidos em diferentes momentos do tempo, até mesmo enquanto a própria empresa ajusta a sua estrutura salarial. Logo, o trabalhador que é contratado entre dois períodos de ajustamento recebe o salário que já está em prática.

\subsection{BARGANHA DE NASH PARA O SALÁRIO}

Neste modelo, firmas e trabalhadores podem se envolver em um processo de barganha cujo resultado é o salário $W_{t}^{*}$. $\eta$ é o poder de barganha dos trabalhadores e $\Delta_{t}\left(W_{i, t}\right)=$ $V_{t}^{E}\left(W_{i, t}\right)-V_{t}^{U}\left(W_{i, t}\right)$ é o superavit da família em ter um trabalhador empregado com salário $W_{i, t}$. Vale ressaltar que, devido à livre entrada nesse mercado, as empresas são economicamente irrelevantes (inutilizáveis) se estão separadas de um trabalhador. $\mathrm{O}$ valor de mercado, $J_{t}\left(W_{i, t}\right)$, de uma empresa que obteve o match corresponde a:

$$
J_{t}\left(W_{i, t}\right)=\Psi_{t}^{L}+E_{t}\left\{\beta_{t, t+1}\left(1-\vartheta_{t+1}\right)\left[\gamma J_{t+1}\left(W_{i, t}\left[\Pi_{t}^{\varepsilon_{w}} \Pi^{1-\varepsilon_{w}}\right]\right)+(1-\gamma) J_{t+1}\left(W_{t+1}^{*}\right)\right]\right\}
$$

Em (15), $\Psi_{t}^{L}$ denota lucros reais (dividendos) por período da firma, os quais dependem do salário, da entrada de trabalho da empresa, da tecnologia vigente, do preço competitivo para o "bem trabalho" em termos reais e de um custo fixo de produção:

$$
\Psi_{t}^{L}=x_{t}^{L} z_{t} h_{i, t}^{\alpha}-\frac{W_{i, t}}{P_{t}} h_{i, t}-\Phi
$$

Para fins de calibração, o custo fixo $\Phi$ é dividido em duas partes: um custo de capital, que beneficia os proprietários da empresa, e um custo fixo de produção, dado em desperdício, logo $\Phi=\Phi^{K}+\Phi^{L}$. Com right-to-manage sobre a negociação salarial, a partir de um certo salário real por hora, as empresas decidem unilateralmente sobre a sua demanda por horas trabalhadas. Cada empresa de bens de trabalho otimiza a demanda até o ponto em que o valor do produto marginal, $x_{t}^{L} m p l_{i, t}$, seja igual ao salário real por hora. Considerando $m p l_{i, t}=z_{t} \alpha h_{i, t}^{\alpha-1}$ para as empresas que entram em barganha, o salário ótimo por hora é obtido por meio do seguinte problema de maximização do excedente entre firmas e trabalhadores:

$$
\arg \max _{W_{i, t}}\left\{\left[\Delta_{t}\left(W_{i, t}\right)\right]^{\eta}\left[J_{t}\left(W_{i, t}\right)\right]^{1-\eta}\right\}
$$




\subsection{ABERTURA DE VAGAS}

A livre entrada no processo de abertura de vagas impulsiona o valor ex ante de uma vaga para zero. Em equilíbrio, o custo real de abertura de uma vaga, $\kappa$, iguala-se ao valor esperado de uma firma de trabalho, devidamente descontados no período $t$, de modo que:

$$
\kappa=q_{t} E_{t}\left\{\beta_{t, t+s}\left[\gamma J_{t+1}\left(W_{i, t}\left[\Pi_{t}^{\varepsilon_{w}} \Pi^{1-\varepsilon_{w}}\right]\right)+(1-\gamma) J_{t+1}\left(W_{t+1}^{*}\right)\right]\right\}
$$

O termo entre colchetes reflete a suposição de que novos postos de trabalho enfrentam os mesmos fatores de rigidez de Calvo que os trabalhadores já existentes, o que é motivado pelas estruturas salariais em empresas multiworker (muitos postos de trabalho) que são ajustadas com baixa frequência. Assim, com probabilidade $(1-\gamma)$, o par empresa-trabalhador pode redefinir o salário no período seguinte, já com a probabilidade $\gamma$ o salário apresenta rigidez e é definido como a média salarial do período anterior.

\subsection{GOVERNO}

A presença do governo representa um ganho fundamental no modelo proposto. Autores como Kato e Miyamoto (2015), Mayer et al (2010) e Gali (2004) reafirmam o poder das contas públicas sobre as oscilações do desemprego, mesmo em economias com percentual baixo de consumo do governo.

O governo recebe receitas provenientes dos impostos lump-sum, $t_{t}$, e das novas emissões de dívida, $D_{t}$. Do lado da despesa, aparece o benefício pago aos desempregados $u_{t} b$, o reembolso do título de dívida pública e os gastos do governo, $g_{t}$. Assume-se também que a política fiscal busca estabilização da dívida. A restrição orçamentária do governo é dada por:

$$
t_{t}+\frac{D_{t}}{P_{t}}=u_{t} b+\frac{D_{t-1}}{P_{t}} R_{t-1}+g_{t}
$$

A autoridade monetária controla a taxa de juros livre de risco dos títulos nominais, $R_{t} . \bar{\Pi}$ é a meta de inflação e $y_{t}^{f l e x}$ é o nível de produto da economia a preços/salários flexíveis (esse é o nível hipotético de produto na ausência de rigidez nominal, tendo os estados na economia como dados) e $\log \left(e_{t}^{\text {money }}\right) \sim$ i.i.d.N $\left(0, \sigma_{\text {monev }}^{2}\right)$ representa um choque de política monetária. A regra de Taylor é tratada na seguinte forma: 


$$
\begin{aligned}
& \log \left(R_{t}\right)=\left(1-\gamma_{R}\right) \log \left(\frac{\bar{\Pi}}{\beta}\right)+\gamma_{R} \log \left(R_{t-1}\right) \\
& +\left(1-\gamma_{R}\right)\left[\frac{\gamma_{\pi}}{4} \log \left(\frac{\Pi_{t}}{\bar{\Pi}^{4}}\right)+\frac{\gamma_{y}}{4} \log \left(\frac{y_{t}}{y_{t}^{\text {flex }}}\right)\right]+\log \left(e_{t}^{\text {money }}\right)
\end{aligned}
$$

A autoridade monetária reage aos desvios da inflação em relação à sua meta trimestral e aos desvios do produto de seu valor a preços e salários flexíveis. Ao calcular essa saída, o sistema acima é duplicado, alterando somente rigidez de preços e salários para zero no modelo flexível.

\subsection{EQUILÍBRIO DE PROCURA}

Finalmente, o fechamento do modelo ocorre quando o produto é esgotado pelo consumo privado $c_{t}$, gastos públicos $g_{t}$, atividade de abertura de vagas $\kappa v_{t}$ e custos fixos de produção dos "bens de trabalho" $n_{t} \Phi^{L}$. Assim, a demanda total é dada por:

$$
c_{t}+g_{t}+\kappa v_{t}+n_{t} \Phi^{L}=y_{t}
$$

\section{RESULTADOS}

\subsection{CALIBRAÇÃO}

O processo de calibração dos parâmetros foi dado com base nos trabalhos de Christoffel, Kuester e Linzert (2009), Attuy (2012) e Moura (2015). Os parâmetros utilizados com mesmo valor dos trabalhos citados são: $\beta$, para a taxa de desconto intertemporal; $\varphi$, para a elasticidade da oferta de horas de trabalho; $\sigma$, para aversão relativa ao risco; $\rho$, para hábito de consumo; $\kappa^{L}$, para desutilidade das horas de trabalho; $\alpha$, para elasticidade trabalho na função produção; $\xi$, para elasticidade desemprego na função matching; $\gamma$ como índice de rigidez salarial ou probabilidade de o trabalhador empregado não entrar em um novo processo de barganha no período seguinte; 9 , para steady state da taxa de separação do mercado de trabalho; $\eta$ para steady state para poder de barganha dos trabalhadores; $\Phi^{K}$ como parcela fixa de retorno do capital; $\Phi^{L}$ como custo fixo associado ao trabalho; $\xi_{w}$ para grau de indexação do salário à inflação; $\varepsilon$ para elasticidade preço da demanda; $\omega$ para fração de firmas que não ajustam preços; 
$\xi_{p}$ como grau de indexação do preço atual à inflação passada; $\gamma_{\pi}$ para elasticidade dos juros em relação à inflação; $\gamma_{y}$ para elasticidade dos juros em relação ao produto; $\gamma_{R}$ para importância que a autoridade monetária dá ao componente inercial da taxa de juros; e $\sigma_{t}^{\text {money }}$ como o desvio-padrão do choque de política monetária.

Conforme Attuy (2012), a taxa de separação do emprego é calibrada como $\vartheta=0,0362$, o poder de barganha dos trabalhadores $\eta$ foi estimado pelo autor em aproximadamente 0,39 para o Brasil e o parâmetro relativo a elasticidade da função matching é $\xi=0,5$. A taxa intertemporal (que mensura a decisão entre poupar ou consumir) também é calibrada seguindo o trabalho de Attuy (2012), no qual o autor utiliza a média das taxas brasileiras trimestrais de juros (Selic) de 1,0362 e inflação (IPCA) de 1,02, condizente com um fator de desconto igual a 0,9803 .

Considerando os parâmetros calibrados, os valores de steady state das variáveis $y$, $u, h$ e $q$ também são calibrados seguindo os mesmos trabalhos. O valor de $y$ no steady state é normalizado em 1 , e $g=0,18$ representa os gastos do governo baseado no estudo de Moura (2015) para a economia brasileira. Além disso, seguindo Attuy (2012), a probabilidade de uma vaga ser preenchida, na economia brasileira, é de cerca de 0,63 , para uma jornada de trabalho equivalente a 8 horas diárias, e a taxa de desemprego próxima de $6,5 \%$. Os demais valores de equilíbrio são encontrados a partir da resolução do sistema de equações no estado estacionário pela própria programação. Destacamos que alguns parâmetros do modelo também são encontrados a partir do equilíbrio do sistema, como $\kappa$ e $\sigma_{m}$.

Tabela 1 - Parâmetros

\begin{tabular}{|c|c|c|}
\hline Parâmetro & Valor & Fonte \\
\hline$\beta$ & 0,9803 & Attuy (2012) \\
\hline$\varphi$ & 2,0000 & Christoffel et al (2009) \\
\hline$\sigma$ & 1,6370 & Moura (2015) \\
\hline$\rho$ & 0,6340 & Moura (2015) \\
\hline$\kappa^{L}$ & 94,7000 & Christoffel et al (2009) \\
\hline$\alpha$ & 0,6500 & Attuy (2012) \\
\hline$\xi$ & 0,5000 & Attuy (2012) \\
\hline$\gamma$ & 0,8300 & Christoffel et al (2009) \\
\hline 9 & 0,0362 & Attuy (2012) \\
\hline$\eta$ & 0,3900 & Attuy (2012) \\
\hline$\Phi^{K}$ & 0,3300 & Christoffel et al (2009) \\
\hline$\Phi^{L}$ & 0,0069 & Christoffel et al (2009) \\
\hline$\xi_{w}$ & 0,0000 & Christoffel et al (2009) \\
\hline$\varepsilon$ & 11,0000 & Attuy (2012) \\
\hline$\omega$ & 0,4770 & Moura(2015) \\
\hline$\xi_{p}$ & 0,0000 & Christoffel et al (2009) \\
\hline$\gamma_{\pi}$ & 1,8920 & Moura (2015) \\
\hline$\gamma_{y}$ & 0,5430 & Moura (2015) \\
\hline$\gamma_{R}$ & 0,6040 & Moura (2015) \\
\hline$\sigma_{t}^{\text {money }}$ & 0,2190 & Moura (2015) \\
\hline
\end{tabular}

Fonte: Elaboração própria. 
Tomando como base os valores de calibração dos parâmetros utilizados acima, busca-se a melhor adequação à realidade da economia brasileira. Na ausência de soluções first-best os valores da literatura internacional foram utilizados.

\subsection{CHOQUE PRINCIPAL: POLÍTICA MONETÁRIA}

O sistema de equações apresentado foi log-linearizado e o modelo DSGE simulado (com a utilização do software MatLab e plataforma Dynare) em busca de funções de impulso resposta das variáveis após um choque de Política Monetária (PM), aplicado na regra de Taylor conduzida pelo Banco Central.

O modelo respeita as condições de Blanchard e Kahn (1980), apresentando equilíbrio estável. O resultados apontam que um choque de política monetária negativo, na escala de $\sigma^{\text {money }}=0,219$, reduz a taxa de juros nominal da economia, o que aumenta diretamente o consumo das famílias, mas afeta a inflação de modo crescente, em proporção inferior ao aumento do custo marginal da economia, em desvios. Com queda na taxa de juros e aumento do consumo, o choque impulsiona o produto final da economia. Esses resultados condizem com a literatura recente, mais especificamente os trabalhos de Valli e Carvalho (2010) e Vereda e Cavalcanti (2010), com uma persistência levemente superior a estes.

Para o mercado de trabalho, as funções impulso resposta (presentes no anexo) condizem com alguns resultados encontrados por Attuy (2012) e Basílio (2012), com persistências superiores dos choques. Em partes, o maior período de persistência pode ser explicado conforme Christoffel, Kuester e Linzert (2009) destacam: pela dinâmica característica do mercado de trabalho, a qual demora um período para que os trabalhadores contratados comecem a exercer suas funções. Esse mecanismo temporal faz com que os choques sejam transmitidos de forma um pouco mais demorada, visto que a absorção não é imediata pelo mercado de trabalho e existem fatores de rigidez presentes no modelo.

A dinâmica ocorre da seguinte forma: devido a um período maturação entre contratação e produtividade do emprego, o número de trabalhadores empregados não pode aumentar instantaneamente. Dessa forma, o ajustamento do trabalho à nova demanda é dado, inicialmente, por um aumento de horas trabalhadas pelos trabalhadores ativos. Mas o aumento da procura também estimula os lucros esperados no setor dos bens de trabalho, que leva sequencialmente a uma maior atividade de abertura de vagas. Como consequência, há mais contratações, de modo que o desemprego cai. Na expectativa de um mercado de trabalho mais aquecido e maiores lucros, o valor de um match existente aumenta e os trabalhadores que renegociam seus contratos ganham salários mais altos. 
A probabilidade de um agente encontrar emprego na economia brasileira aumenta consideravelmente, mas a chance de uma nova vaga ser preenchida cai, pois o número de vagas aumenta de maneira proporcionalmente maior em relação aos novos matches da economia, dada a tecnologia de informação desse processo. Sequencialmente, a mudança de salários e no produto marginal do trabalho, conjuntamente, implicam no aumento da inflação.

Por fim, o processo de barganha gera um valor de salário final levemente superior, devido ao aquecimento do consumo que induz maior demanda das empresas pela mão de obra, proporcionando certa vantagem às famílias no processo de negociação final. Já o superavit da família em ter um trabalhador empregado ao salário de barganha cai, provavelmente devido ao aumento da jornada de trabalho e à corrosão do salário vigente com avanço da inflação. Ainda, o valor de mercado da firma com um novo match cai expressivamente, principalmente por conta do aumento no salário final pago (após a barganha) e da redução no lucro, dado o avanço no custo marginal.

\subsection{CHOQUES ADICIONAIS}

Com objetivo de dar robustez ao modelo de estudo e contribuir para o atual debate de políticas econômica e desemprego no cenário brasileiro, choques positivos de gastos do governo e produtividade são explorados. Para execução desses choques utilizamos os desvios-padrão estimados por Moura (2015): $\sigma^{z}=0,192$ e $\sigma^{g}=0,062$. Os possíveis efeitos no mercado de trabalho são comentados a seguir, todas as funções impulso-resposta estão presentes no anexo.

\subsubsection{CHOQUE DE GASTOS DO GOVERNO}

O choque de gastos públicos segue o padrão da literatura para as principais variáveis macroeconômicas (conforme Valli e Carvalho, 2010; Vereda e Cavalcanti, 2010). O excesso de gastos do governo eleva a inflação e gera um aumento da taxa de juros nominal da economia, o que reduz diretamente o consumo das famílias. Contudo, o poder do choque de gastos na economia brasileira é alto a ponto de impulsionar o produto final por um tempo muito superior comparado à política monetária (principalmente por meio do canal com mercado de trabalho), mesmo com uma queda no consumo.

O ponto principal na dinâmica do choque positivo de gastos do governo no mercado de trabalho é em relação à duração dos efeitos sobre as variáveis. Ocorre um aumento inicial do número de horas trabalhadas e um aumento posterior do emprego, 
novamente devido ao período de maturidade dos contratados. Contudo, no caso da política fiscal, parece que a redução no desemprego aparenta ser mais prolongada. Basicamente, o número de matchs é positivamente afetado com um pouco menos de intensidade, mas as vagas geradas aquecem a economia por um período de tempo bem superior.

Ainda em relação ao mercado de trabalho, a probabilidade de um agente encontrar emprego na economia brasileira aumenta, mas a chance de uma nova vaga ser preenchida cai, pois o número de vagas aumenta de maneira proporcionalmente maior em relação aos novos matches da economia, contudo esses movimentos perduram por mais tempo no caso da expansão de gastos do governo. Já em relação ao processo de barganha, o valor do salário final cai (em oposição ao choque negativo de política monetária), muito por conta da redução no consumo e nos avanços da inflação, mesmo com a maior demanda das empresas pela mão de obra. Já o superavit da família em ter um trabalhador empregado ao salário de barganha aumenta (provavelmente devido ao prolongado deslocamento da demanda por mão de obra advinda do fomento governamental) e o valor de mercado da firma com um novo match também aumenta a expressivamente devido à queda no salário final de barganha e ao aumento do lucro.

\subsubsection{CHOQUE DE PRODUTIVIDADE}

Em sequência, o choque tecnológico também ocorre conforme o esperado para o caso brasileiro, mas de forma mais intensa e duradoura do que os choques de política monetária e política fiscal. O produto apresenta uma resposta crescente e as horas trabalhadas caem com aumento da produtividade. Essa nova oferta provoca uma redução de preços, necessária para que a produção seja absorvida por meio do aumento no consumo final das famílias. Como consequência, ocorre uma redução na taxa de juros para contrabalancear o aumento no produto e queda na inflação.

No mercado de trabalho também ocorre uma queda bem mais expressiva e duradoura do desemprego e das horas trabalhadas. Essa queda ocorre pela abertura de vagas e crescimento de matches na economia, fazendo com que a probabilidade de um agente encontrar emprego na economia brasileira aumente, mas a chance de uma nova vaga ser preenchida diminua. Esses movimentos, além de apresentarem maior desvio, também afetam a economia por um período de tempo superior.

Finalmente, em relação ao processo de barganha, observamos que valor do salário final, o superavit da família em ter um trabalhador empregado ao salário de barganha e o valor de mercado da firma com um novo match aumentam. Aqui destacamos um efeito novo, que eleva todos os elementos de processo de barganha conjuntamente. 
Isso ocorre, majoritariamente, pelo canal de substituição tecnológica, que exige maior especialização do trabalhador e implicaria em remunerações superiores. Logo, além de melhorar o superavit das famílias, a nova tecnologia alavanca mais do que proporcionalmente o lucro das firmas.

\subsection{ANÁLISE DE DESVIOS-PADRÃO}

Buscando identificar se o modelo proposto ajuda a compreender a realidade do mercado de trabalho e da economia brasileira, os desvios-padrão e a correlação do modelo simulado serão comparados com as séries históricas de produto e desemprego. Essa escolha ocorre por dois motivos: i) o desemprego e o produto serem as variáveis mais utilizadas na literatura search como medida para análise de ajustamento dos modelos; ii) devido à inexistência de dados para a economia brasileira, de muitas das variáveis agregadas do mercado de trabalho, problema que também é destacado por Attuy (2012).

Uma análise de sensibilidade aos desvios também será feita com base na oscilação do parâmetro $\gamma$ (índice de rigidez salarial, que reflete a probabilidade de o trabalhador empregado conseguir entrar em um novo processo de barganha), que, na ausência de valores para o Brasil, foi calibrado em 0,83, conforme o estudo de Christoffel, Kuester e Linzert (2009) para a União Europeia, ou seja, tal calibração não necessariamente reflete as características do mercado de trabalho brasileiro.

Como o modelo é simulado a partir de log-desvios, faz-se necessária a manipulação das séries para comparação dos desvios. O processo foi executado da seguinte forma: as séries de PIB e Desemprego, coletadas no IBGE (Contas Nacionais e Pesquisa Mensal do Emprego), com periodicidade mensal de 2003:01 a 2016:02², foram dessazonalizadas e trimestralizadas (via média simples). Em seguida, foi aplicado o logaritimo neperiano nos dados históricos e, por fim, visando à remoção da tendência, os dados foram submetidos a três processos de filtragem, buscando dar maior robustez aos resultados: i) filtro de Hodrick-Prescott - consideramos o parâmetro do filtro $\lambda=1600$, mais adequado para periodicidade trimestral dos dados; ii) filtro de Baxter-King - a literatura sugere um default entre 8 e 12 para o parâmetro de truncagem, e escolhemos $\mathrm{k}=8$ devido à frequencia trimestral e ao tamanho da série, já que se faz necessária uma remoção de $2 \mathrm{k}$ dados ( $\mathrm{k}$ em cada ponta), e quanto aos limites dos

2 Vale ressaltar que em 2001 o IBGE executou um amplo processo de revisão metodológica na série de desemprego, buscando melhor adequação às características do trabalho e às formas de inserção da população ocupada. Logo, os resultados encontrados para o período considerado não são afetados por mudanças metodológicas, dado que a partir de novembro de 2002 o sistema de cálculo da série foi unificado. 
ciclos, escolhemos os valores de 2 até 20 trimestres, com base no relatório do Comitê de Datação de Ciclos Econômicos CODACE-FGV (FGV, 2015); iii) decomposição de Beveridge e Nelson - nesse caso, utilizamos o critério de autosseleção ARIMA para adequação do processo de filtragem e remoção dos ciclos. A Tabela 2 apresenta os desvios padrão e correlações:

Tabela 2 - Desvios-Padrão e Correlação

\begin{tabular}{ccccccc}
\hline Variável & Filtro HP & Filtro BK & Filtro BN & $\gamma=\mathbf{0 , 9 3}$ & $\begin{array}{c}\text { Modelo } \\
\text { Calibrado } \\
\text { Básico }\end{array}$ & $\gamma=\mathbf{0 , 7 3}$ \\
\hline $\begin{array}{c}\text { Desvio Padrão } \\
y_{t}\end{array}$ & $\mathbf{1 , 7 7}$ & $\mathbf{1 , 5 3}$ & $\mathbf{1 , 3 2}$ & 2,07 & 1,84 & 1,68 \\
$u_{t}$ & $\mathbf{8 , 8 3}$ & $\mathbf{8 , 4 9}$ & $\mathbf{9 , 1 0}$ & 12,99 & 10,95 & 8,85 \\
$u_{t} / y_{t}$ & $\mathbf{4 , 9 8}$ & $\mathbf{5 , 5 4}$ & $\mathbf{6 , 8 9}$ & 6,27 & 5,95 & 5,26 \\
$\begin{array}{c}\text { Correlação } \\
{\left[y_{t} u_{t}\right]}\end{array}$ & $\mathbf{- 0 , 7 6 5}$ & $\mathbf{- 0 , 6 8 7}$ & $\mathbf{- 0 , 7 0 4}$ & $-0,886$ & $-0,869$ & $-0,834$ \\
\hline
\end{tabular}

Fonte: Elaboração própria.

Segundo as simulações e os dados trabalhados, parece que o modelo desenvolvido se ajusta relativamente bem à série de desemprego da economia brasileira. Existem indícios de que a probabilidade de o trabalhador empregado conseguir entrar em um novo processo de barganha seria menor, o que agrava o processo de rigidez salarial no Brasil. Logo, comparando ao valor europeu utilizado no modelo simulado, a análise de sensibilidade indica que para valores inferiores desse parâmetro os desvios relativos e a correlação se ajustam melhor às séries históricas de interesse.

\section{CONCLUSÃO}

A construção/simulação de modelos DSGE para o Brasil ainda é escassa, principalmente no que se refere à análise de como os impactos de fricções no mercado de trabalho afetam as oscilações econômicas dados choques exógenos. Este trabalho buscou contribuir com a literatura, apresentando um modelo DSGE aplicável ao caso brasileiro, com fricções do tipo searching and matching e barganha de Nash.

Procurou-se simular um modelo dinâmico e estocástico de equilíbrio geral baseado em Christoffel, Kuester e Linzert (2009), calibrando os parâmetros estruturais específicos de acordo com a literatura brasileira e explorando uma análise de impulso-resposta sobre o equilíbrio econômico no país (principalmente sobre as variáveis do mercado de trabalho), dado um choque de política monetária para um regra de Taylor baseada na diferença do produto e seu nível a preços flexíveis, com meta de inflação anualizada. 
Os resultados da simulação apontam que para um choque de política monetária ocorre uma queda nos juros nominais, aquecendo o produto da economia pelo lado da demanda por meio do crescimento do consumo das famílias. Logo, as firmas passam a contratar mais mão de obra, aquecem o mercado de trabalho reduzindo o desemprego e pressionam o salário de equilíbrio para cima, após o processo de barganha, o que contrabalanceia o efeito sobre os custos marginais na economia e conduz a um aumento do nível de preços.

Inicialmente, dentro do mercado de trabalho ocorre um aumento da jornada de trabalho em busca de suprir as novas demandas. Em sequência, o número de matches cresce devido ao crescimento expressivo de vagas disponíveis na economia, dado o aquecimento do consumo. Assim, passa a existir uma maior probabilidade de um agente encontrar emprego na economia brasileira, mas uma queda na chance de uma nova vaga ser preenchida.

As imperfeições informacionais consideráveis no mercado de trabalho brasileiro, as quais levam o número de vagas a aumentar proporcionalmente mais do que o número de novos matches alcançados na economia, não permitem que o desemprego caia ainda mais no curto prazo, dada a quantidade de vagas que ficam ociosas em relação ao trabalhadores disponíveis, seja por questões informacionais ou até mesmo de qualificação. Já para o processo de barganha de Nash, o modelo aponta que para o choque dado o superavit da família em ter um trabalhador empregado ao salário de barganha cai devido ao aumento da jornada de trabalho. Além disso, o valor de mercado da firma, com um novo match, também cai, mas para o meio do avanço nos custos de produção, pressionados pelo aumento salarial.

Para dar robustez à análise foram executados choques adicionais (de gastos governamentais e produtividade), uma análise de sensibilidade e comparação dos desvios-padrão simulados com os dados históricos das séries brasileiras de produto e desemprego. Tais procedimentos confirmam a adequação dos resultados com o que é descrito pela literatura e apresentam indícios de que a probabilidade de o trabalhador empregado conseguir entrar em um novo processo de barganha seja inferior ao imaginado. Logo, os salários se manteriam mais rígidos e existiria uma maior dificuldade (estrutural) nos esforços do trabalhador brasileiro por rendimentos maiores a cada período, comparativamente ao valor base utilizado.

Um complemento do modelo com subtipos de família (ricardianas e Rule of Thumb), além de uma modelagem para o setor externo e taxa de câmbio, inclusão de restrições ao crédito ou até mesmo alteração do tipo de rigidez utilizada podem levar a resultados diferentes quanto à magnitude dos desvios. No entanto, mesmo de forma simples e com algumas limitações, o modelo aparenta levar a conclusões relevantes sobre a interação entre fricções do mercado de trabalho e oscilações da economia bra- 
sileira, condizentes com os trabalhos de Basílio (2012) e Attuy (2012) e com as séries de dados trabalhadas.

\section{REFERÊNCIAS}

ADJEMIAN, S. ET AL. Dynare: reference manual, Version 4. Dynare Working Papers, n. 1, CEPREMAP, 2011.

ANDOLFATTO, D. Business cycles and labor-market search. The American Economic Review, v. 1, n. 86, p. 112-132, 1996.

ATTUY, G. Ensaios sobre macroeconomia e mercado de trabalho. Tese (Doutorado em Economia) - Faculdade de Economia e Administração, Universidade de São Paulo, São Paulo, 2012.

BASÍLIO, F. A. C. Política monetária e barganha salarial: a importância das instituições do mercado de trabalho na ausência de rigidez nominal. Tese (Doutorado em Economia) - Universidade de Brasília, Brasília-DF, 2012.

BLANCHARD, O. J.; KAHN, C. M. The solution of linear difference models under rational expectations. Econometrica, v. 48, n. 5, p. 1305-11, 1980.

CHRISTOFFEL, K.; KUESTER, K.; LINZERT, T. The Role of labor markets for euro area monetary policy. European Economic Review, n. 53, p. 908-936, 2009.

FGV - FUNDAÇÃO GETÚLIO VARGAS. Comunicado do Comitê de Datação de Ciclos Econômicos (CODACE) - Instituto Brasileiro de Economia (IBRE), Rio de Janeiro, Ago. 2015. Disponível em: <http://portalibre.fgv.br/lumis/portal/file/fileDownload.jsp?fileId=8A7C8 2C54DB5CA9F014EF8794F847120> Acesso em: 10 jun. 2017.

DENHANN, R.; WATSON. Job destruction and propagation of shocks. American Economic Review, v. 90, n. 3, p. 482-498, 2000.

DIAMOND, P. A. Wage determination and efficiency in search equilibrium. Review of Economic Studies, v. 49, n. 2, p. 217-227, 1982a.

DIAMOND, P. A. Aggregate demand management in search equilibrium. The Journal of Political Economy. v. 90, n. 5, p. 881-894, 1982b.

GALI, J.; LOPEZ-SALIDO, J. D; VALLES, J. Understanding the effects of government spending on consumption. IMF Working Papers, n. 339, April, 2004.

GERTLER, M.; TRIGARI A. Unemployment fluctuations with staggered nash wage bargaining. Journal of Political Economy, v. 117, n. 1, p. 38-86, 2009.

HALL, R. E. Employment fluctuations with equilibrium wage stickness. American Economic Review, v. 95, n. 1, p. 50-64, 2005.

IBGE - INSTITUTO BRASILEIRO DE GEOGRAFIA E ESTATÍSTICA. Séries estatísticas. [On-line] IBGE. Disponível em: <http://seriesestatisticas.ibge.gov.br/>. Acesso em: 25 set. 2015.

KATO, R. R; MIYAMOTO, H. Effects of fiscal stimulus on the labor market. Public Policy Review, Policy Research Institute, Ministry of Finance, Japan, v. 11, n. 2, Mar. 2015. 
MAYER, E.; MOYEN, S.; STAHLER, N. Government expenditures and unemployment : a DSGE perspective. Discussion Paper, Deutsche Bundesbank, Series 1, Economic Studies, 2010.

MORTENSEN, D. T. "The matching process as a noncooperative bargaining game". In: MCCALL, J. J. (Org.). The economics of information and uncertainty. Chicago: University of Chicaco Press, 1982, p. 233-254.

MORTENSEN, D.; PISSARIDES, C. A. Job creation and job destruction in the theory of unemployment. Review of Economic Studies, v. 61, n. 3, p. 397-415, 1994.

MOURA, G.V. Multiplicadores fiscais e investimento em infraestrutura. Revista Brasileira de Economia, v. 69, n. 1, 2015.

PETRONGOLO, B.; PISSARIDES, C. Looking into the black box: a survey of the matching function. Journal of Economic Literature, v. 39, n. 2, p. 390-431, 2001.

PISSARIDES, C. Short-run equilibrium dynamics of unemployment, vacancies, and real wages. American Economic Reivew, v. 75, p. 676-690, 1985.

SHIMER, R.; SMITH, L. Matching, search, and heterogeneity. Advances in Macroeconomics, v. 1, n. 1, 2001.

VALLI, M.; CARVALHO, F. Fiscal and monetary policy interaction: a simulation based analysis of a two-country New Keynesian DSGE model with heterogeneous households. Working Paper, Banco Central do Brasil, n. 204, 2010.

VEREDA, L.; CAVALCANTI, M. A. F. H. Modelo dinâmico estocástico de equilíbrio geral (DSGE) para a economia brasileira: Versão 1. Texto para Discussão, IPEA, n. 1479, 2010.

\section{ANEXO - GRÁFICOS IMPULSO RESPOSTA}

\section{Figura 1 - Impulso Resposta - Choque de Política Monetária}
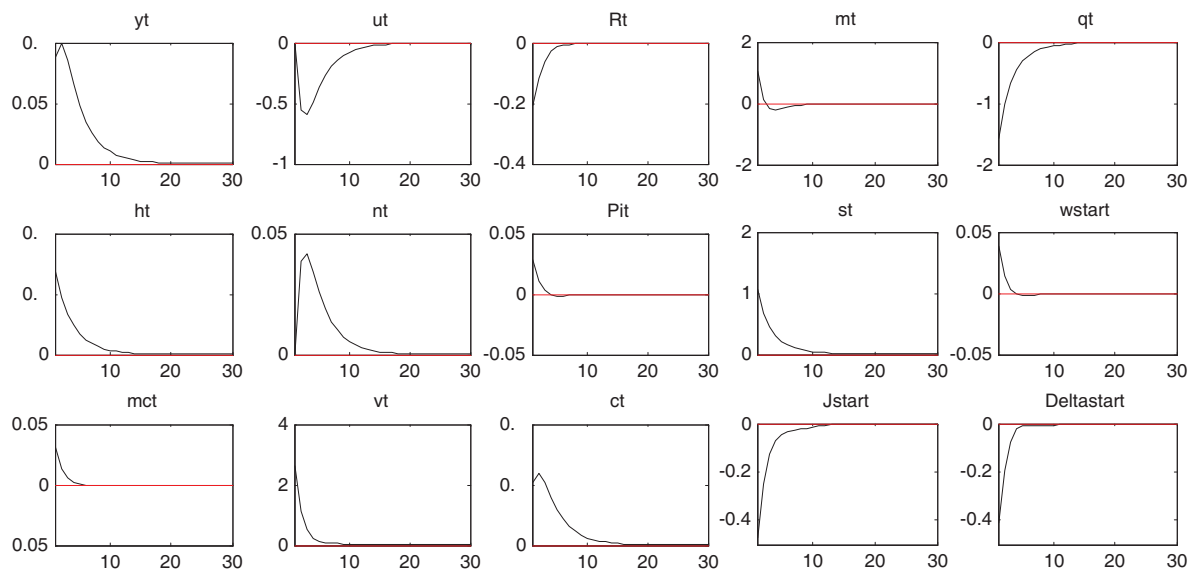

Fonte: Elaboração própria. 
Figura 2 - Impulso Resposta - Choque de Gastos do Governo
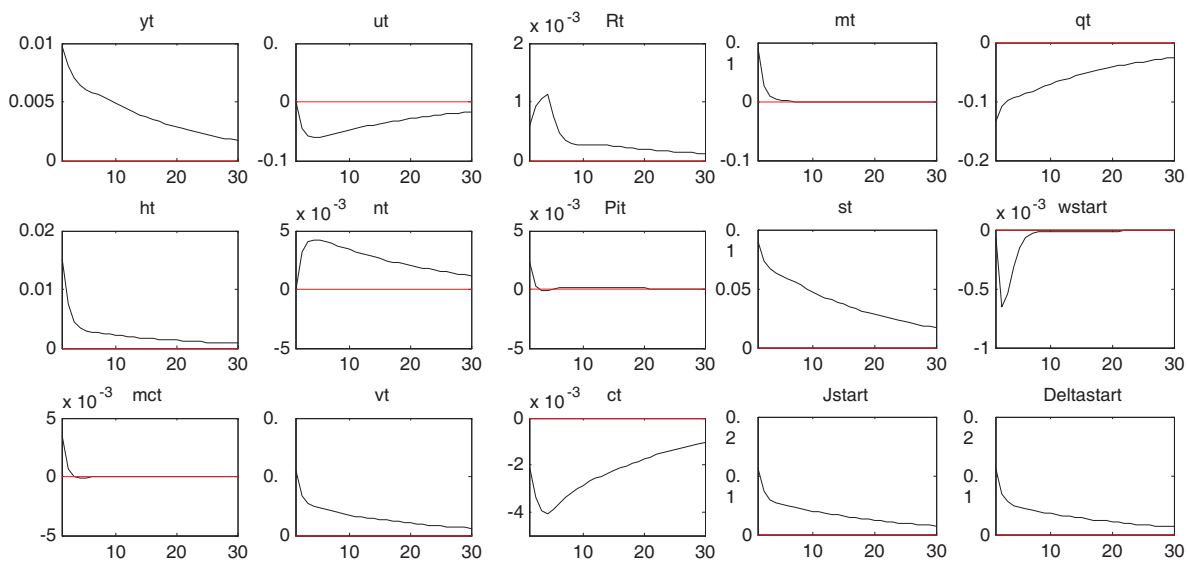

Fonte: Elaboração própria.

Figura 3 - Impulso Resposta - Choque de Produtividade
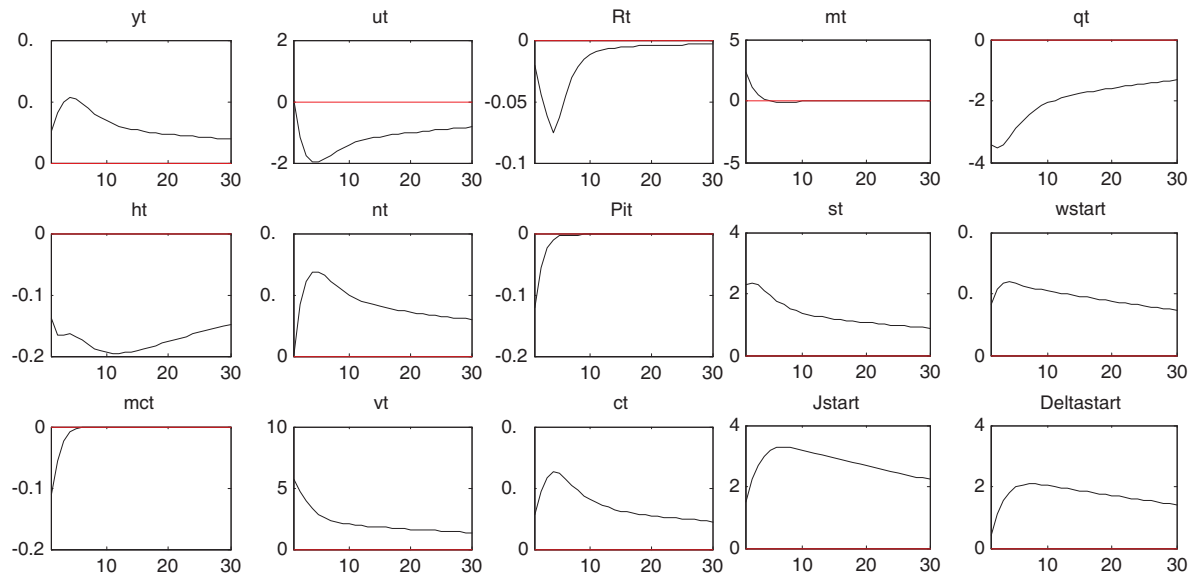

Fonte: Elaboração própria. 